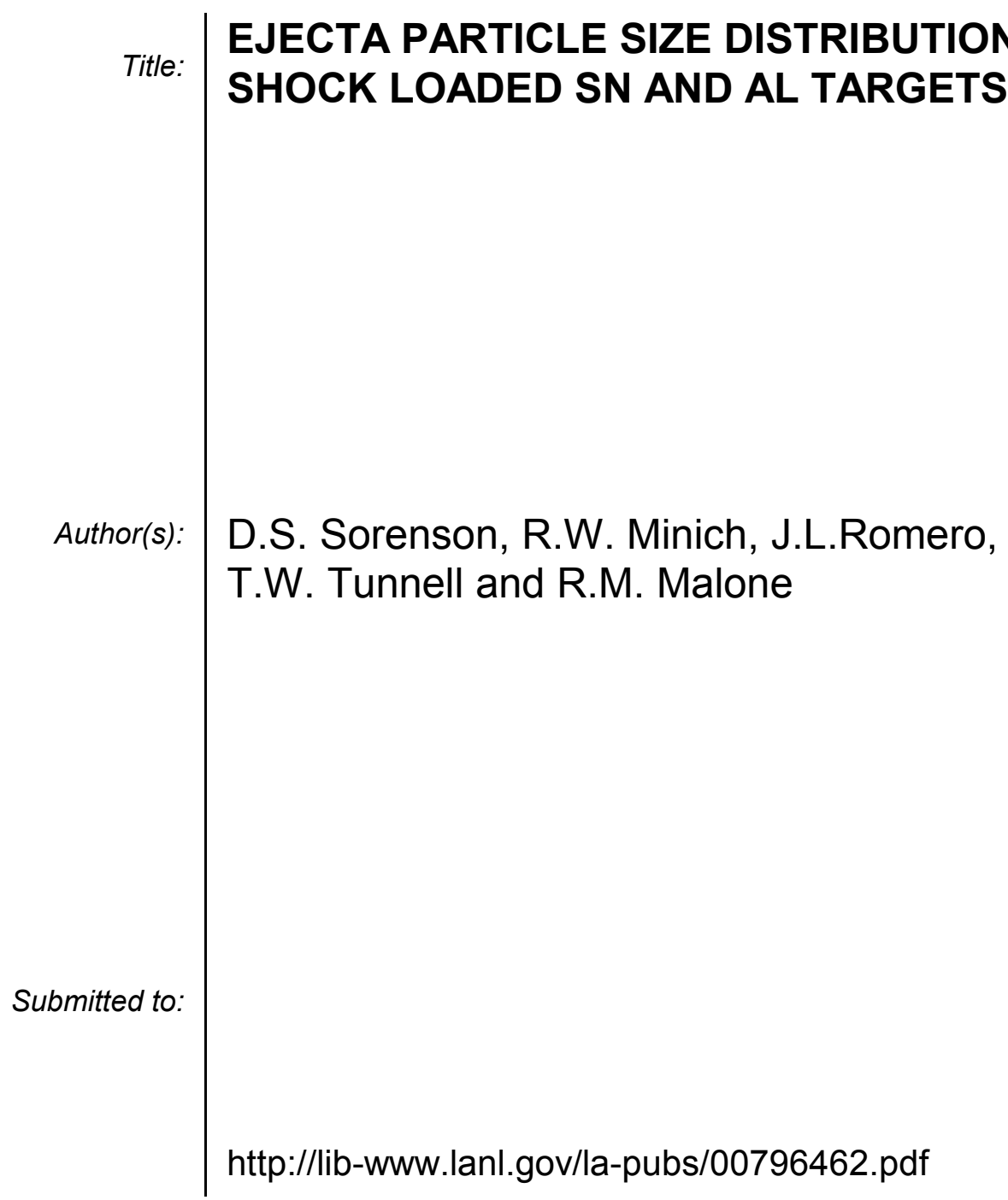

Los Alamos National Laboratory, an affirmative action/equal opportunity employer, is operated by the University of California for the U.S. Department of Energy under contract W-7405-ENG-36. By acceptance of this article, the publisher recognizes that the U.S. Government retains a nonexclusive, royaltyfree license to publish or reproduce the published form of this contribution, or to allow others to do so, for U.S. Government purposes. Los Alamos National Laboratory requests that the publisher identify this article as work performed under the auspices of the U.S. Department of Energy. Los Alamos National Laboratory strongly supports academic freedom and a researcher's right to publish; as an institution, however, the Laboratory does not endorse the viewpoint of a publication or guarantee its technical correctness. 


\title{
EJECTA PARTICLE SIZE DISTRIBUTIONS FOR SHOCK LOADED SN AND AL TARGETS
}

\author{
D.S. Sorenson ${ }^{1}$, R.W. Minich ${ }^{2}$, J.L.Romero ${ }^{3}$, T.W. Tunnell ${ }^{4}$, R.M. Malone ${ }^{4}$ \\ ${ }^{1}$ Los Alamos National Laboratory, Los Alamos, NM 87544 \\ ${ }^{2}$ Livermore National Laboratory, Livermore, CA 94550 \\ ${ }^{3}$ University of California, Davis, CA 95616 \\ ${ }^{4}$ Bechtel Nevada, Los Alamos, NM 87544
}

\begin{abstract}
When a shock wave interacts at the surface of a metal sample "ejected matter" (ejecta) can be emitted from the surface. The mass, size, shape, and velocity of the ejecta varies depending on the initial shock conditions and the material properties of the target. To understand this phenomena, experiments have been conducted at the Pegasus Pulsed Power Facility (PPPF) located at Los Alamos National Laboratory (LANL). The facility is used to implode cylinders to velocities of many $\mathrm{mm} / \mu \mathrm{sec}$. The driving cylinder impacts a smaller target cylinder where shock waves of a few hundreds of kilobars can be reached and ejecta formation proceeds. The ejecta particle sizes are measured for shock loaded Sn and Al metal samples using an in-line Fraunhofer holography technique. The distributions will be compared to calculations from 3 and 2 dimensional percolation theory.
\end{abstract}

\section{INTRODUCTION}

Metals under shock-loaded conditions can lead to complex phenomena depending on the properties of the material and initial shock conditions. The phenomena being reported here involves particle ejection which results from a shock wave interacting at a metal vacuum (gas) interface. For these experiments, particle sizes are measured using holography. Ejecta experiments similar to these have been performed at other facilities $[1,2,3,4,5]$ however, only a few measurements of particle sizes have been carried out. The experiments were performed at the Pegasus Pulsed Power Facility (PPPF) [6,7] at Los Alamos National Laboratory. Particle size distributions resulting from microjet production will be presented for shock loaded $\mathrm{Al}$ (6061 T-6) and Sn metal samples. The shock pressures generated in the samples were 300 and $400 \mathrm{kbar}$ respectively. The measured particles size distributions will be compared with predictions from percolation theory.
Many phenomena in nature involve fragmentation from very small (nuclei) to large (planets) scale systems $[8,9,10,11]$. A useful model that has been used to describe the fragment size distributions is based on percolation theory. For example, fragmentation of heavy nuclei resulting from high-energy nucleus-nucleus interactions $[9,10]$ are described well using this theory. Percolation theory predicts simple power laws with values that depend on the dimensionality of the system being investigated. For example, the powers change depending if the system is one, two or three-dimensional [12] and thus provide insight into the dimensionality of the system being investigated. We will use predictions from percolation theory in the interpretation of the data.

\section{EXPERIMENTAL PROCEDURE}

For the $\mathrm{Al}$ and $\mathrm{Sn}$ experiments, a 400-micron thick target cylinder measuring $3.0 \mathrm{~cm}$ in diameter is 
impacted with another cylinder producing a pressure of $300 \mathrm{kbar}$ for the $\mathrm{Al}$ experiment and $400 \mathrm{kbar}$ for the Sn experiment. In both cases, the metal surface was prepared initially with a surface finish of $20 \mu$ " $\mathrm{R}_{\mathrm{a}}$. Grooves were subsequently machined into the target at various locations azimuthally around the target. Fig. 1 shows a cross section of these grooves which were machined into the $\mathrm{Al}$ and $\mathrm{Sn}$ cylinder at two depths of 20 and 100 (not shown) microns and groove angles of 60 and 120 degrees. The grooves establish the initial surface conditions for the formation of microjets and the subsequent fragmentation. In addition, the grooves allow some control over the amount of energy available for the

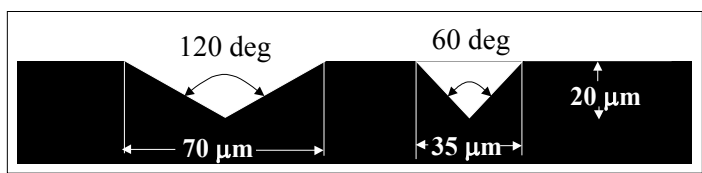

Figure 1. Cross section of grooves that were machined into the $\mathrm{Al}$ and $\mathrm{Sn}$ cylindrical targets

fragmentation process. This is manifested in the increased microjet velocities for smaller groove angles.

For these experiments, the impacting cylinder is used to set up the initial shock wave conditions in the metal sample. The resulting shock wave that is setup in the metal sample then interacts at the Al (Sn)-vacuum interface producing microjets at the groove locations. The microjets produced then pass through a 1.5 diameter tantalum cylindrical mask. The measurement of the particles is carried out using an in-line Fraunhofer holography technique $[13,14,15]$ which records a 3 dimensional image of the particles over a cylindrical volume $15 \mathrm{~mm}$ in diameter and $6 \mathrm{~mm}$ long. The particle distributions are later reconstructed [16] from the hologram. Resolution of the system is approximately 1.5 microns.

\section{RESULTS FOR SHOCK LOADED AL AND SN.}

Fig. 2 shows an image of the hologram (viewed as a shadowgram) obtained from the shocked Al experiment. The holographic film records the phase information used to reconstruct the ejecta particles, and a DC term which forms a shadowgram of the microjets. The shadowgram of the ejecta and the various calibration markers are clearly observable in the figure, whereas the interference patterns used in the reconstruction of the individual particles are not visibly discernable. The figure is viewed into the source of the laser beam where the outer part of the image corresponds to just inside the $1.5 \mathrm{~cm}$ diameter collimator. The figure shows micro jets originating from two groove angles of 60 and 120 degrees. The microjets from the 120

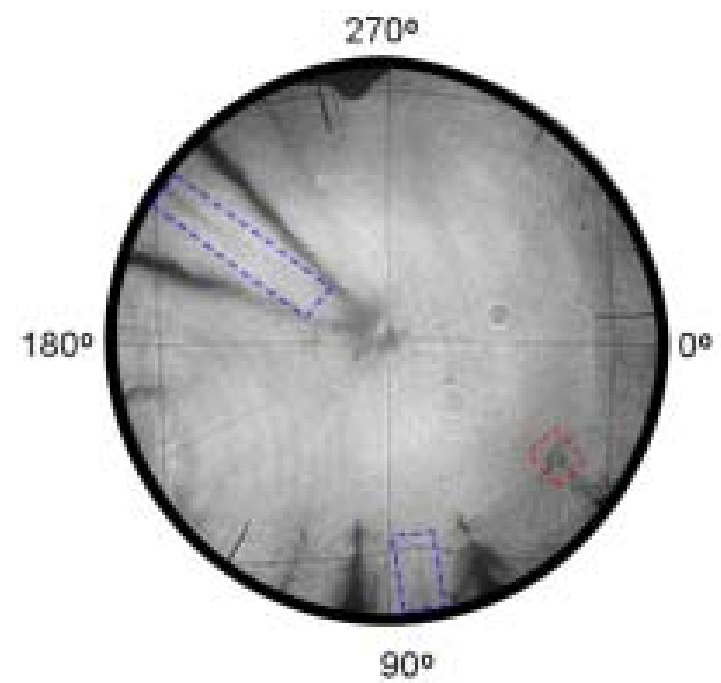

Figure 2. Image of hologram viewed as a shadowgram. 12 mciron diamter calibration wires are shown along with 75 micron diamter pegs at 125,255 , and 355 degrees. Microjets produced from the 60 and 120 degree grooves are clearly seen.

degree $\mathrm{V}$ grooves span the angular region shown on the figure from 7.5 degrees to 112.5 degrees incrementing every 15 degrees. The 60 degrees V grooves span the angular range 137.5 through 242.5 degrees incrementing every 15 degrees. Also, clearly shown in Fig. 2 are the three posts at 125, 255, and 355 degrees marking the center axial position of each masked region. In addition, six 12 micron diameter tungsten wires are strung in the collimator for further calibration. 


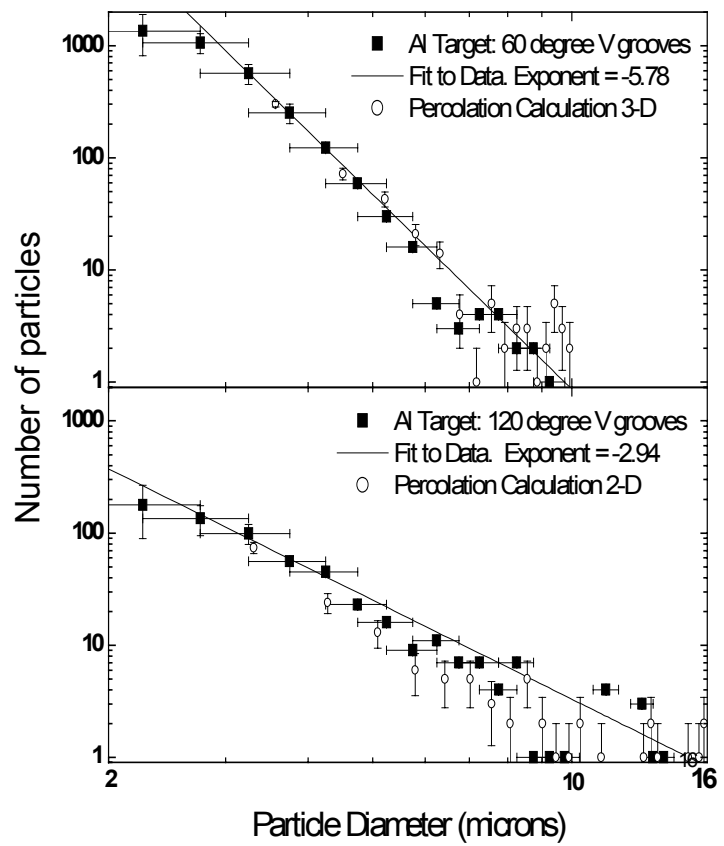

Figure 3. Particle size distributions for $\mathrm{Al}$ experiment. Top plot is for 60 degree $\mathrm{V}$ groove, and bottom is for 120 degree $\mathrm{V}$ grooves. Solid curves are fits to the data using a power law. Percolation calculations are shown as the open circles, and the data is shown as the solid squares.

The $\mathrm{Al}$ ejecta particles distributions are shown in Fig. 3 for the 60 and 120 microjets. The shape of the distribution can be fit well using a power law function. The powers that fit the data are -5.78 for the 60 degree microjets, and -2.94 for the 120 degree microjets. The error bars shown are due to two sources. The first source of error has to do with finite resolution in the reconstruction system and noise associated with the recording of the hologram and reconstructing the data. This error is typically $+/-0.5$ micron. The second error has to do with the low signal to noise ratio that is encountered at our resolution limit between 1.5 and 2.5 microns in diameter. Also shown in the figure are percolation calculations shown as the open circles. The calculation in the top figure was done using a 3-D cubic lattice, whereas the bottom figure required using a 2-D lattice. Both calculations were performed at the critical probabilities of 0.25 and 0.5 respectively. The dramatic change in the slope for the two cases suggests that the physics for the fragmentation may be quite different. In terms of percolation theory, the 60 degree $\mathrm{V}$ groove data is

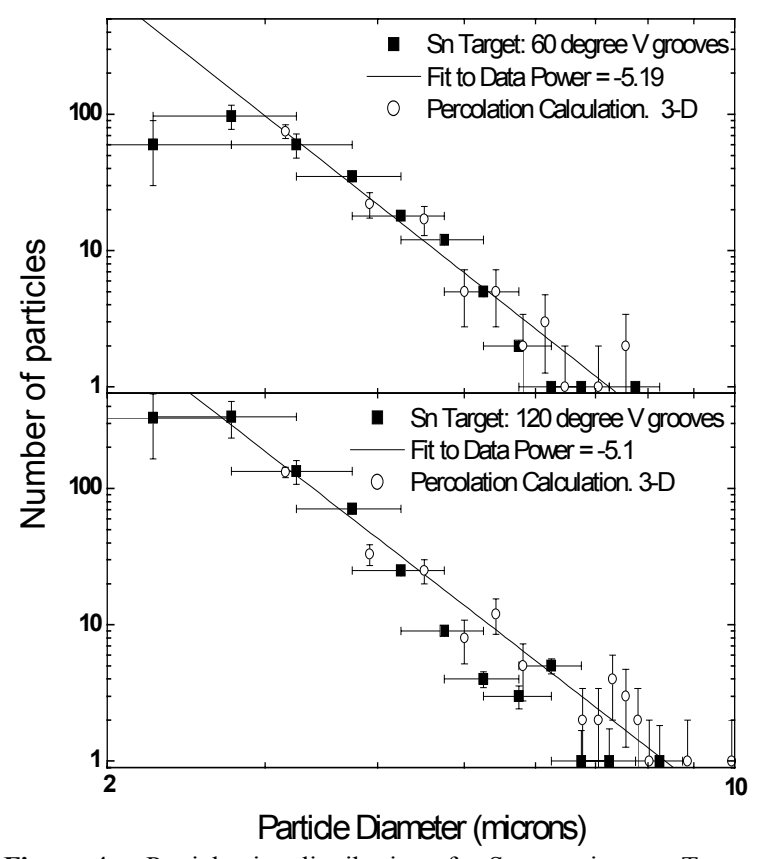

Figure 4. Particle size distributions for Sn experiment. Top plot is for 60 degree $\mathrm{V}$ grooves, and bottom is for 120 degree $\mathrm{V}$ grooves. Solid curves are fits to the data using a power law. Percolation calculations are shown as the open circles, and the data is shown as the solid squares.

consistent with a 3-D breakup, whereas the 120 degree $\mathrm{V}$ groove data is undergoing a 2-D breakup. Furthermore, since the percolation calculations were done at the critical probability, this suggests the metal may be near a phase transition.

Fig. 4 shows the particle distributions for the shocked Sn experiment. The initial metal surface conditions were identically to the Al case, but the shock pressure in the Sn sample was 400 kbar. With this shock pressure, it was calculated to be well above melt conditions. The distributions shown in the figure both are fit well with a power close to -5 . The data agrees well with 3-D percolation calculations at the critical probability

\section{DISCUSSIONS AND CONCLUSIONS}

In this report we have presented ejecta particle size distributions for shock loaded $\mathrm{Sn}$ and $\mathrm{Al}$ samples. The measurements were accomplished using an in-line Fraunhofer holography technique. Grooves were machined into the target sample producing well characterized initial conditions for the 
production of microjets. The microjets fragment giving rise to particle distributions that were measured holographically. Within the context of percolation theory, we have fit these ejecta particle distributions with both 3-D and 2-D models. The Al data shows a dramatic difference in the distribution shapes going from the 60 to 120 degree $\mathrm{V}$ grooves. This we understand to be consistent with the difference between a 3-D and 2-D fragmentation process. Possibly, the $\mathrm{Al}$ in the latter case is undergoing spallation. The Sn data for both grooves gives very similar shapes for the distributions consistent with 3-D fragmentation. The implications of these results are being further investigated

\section{ACKNOWLEDGMENTS}

The authors would like to acknowledge B. C. Froggett, R.L. Flurer, and J. Roberts who ensured that the optical relay system and laser systems were properly setup for recording the hologram. The target assemblies were provided and built by W.E. Anderson, J. Bartos, and the rest of the fabrication team. In addition, many people were involved in setting up and recording data. These included D.T. Westley, C.Y. Tom, G. Allred, as well as the facility operations support team at the Pegasus Pulsed Power Facility headed by J. Cochrane, D.W. Scudder, and R. R. Bartsch. Other measurements were done to determine the dynamics of the target assembly. These were carried out by D.V. Morgan, D. Platts, J. Stokes, B. Broste, P. Rodriguez, and L. Veeser. We also acknowledge theoretical support from H. Lee, M. G. Sheppard, R.L. Bowers, R. Gore, G. Bazan, and A. J. Scannapieco. This work was supported by the High Energy Density Program and funded by the United States Department of Energy.

\section{REFERENCES}

1. P. Andriot, P. Chapron, V. Lambert, F. Olive, "Influence of Melting on Shocked Free Surface
Behavior using Doppler Laser Interferometry and XRay Densitometry," Shock Waves in Condensed Matter, Elsevier Science Publishers B.V., p. 277 (1983).

2. J.R. Assay, "Material Ejection from Shock-Loaded Free Surfaces of Aluminum and Lead," Sandia Laboratories Report SAND 76-0542 (1976) (unpublished).

3. P. Elias, P. Chapron, M. Mondot, "Experimental study of the slowing down of shock-induced matter ejection into argon gas." Shock Compression in Condensed Matter 1989, Elsevier Science Publishers B.B., p. 783, (1990).

4. C. McMillan, R. Whipley, Proceedings of the 18th International Congress on High-Speed Photography and Photonics, Xian, China (1988).

5. C.F. McMillan, "Size measurements of high velocity particle distributions," SPIE 674 High Speed Photography (Pretoria) 289 (1986).

6 M. P. Hockaday et. al., "Liner Target Interaction Experiments on Pegasus II"'" Proceedings of the 10th IEEE Pulsed Power Conference, Albuquerque, NM,1995, p. 586.

7 R.L. Bowers et. al., " Precision Solid Liner Experiments on Pegasus II", Proceedings of the 10th IEEE Pulsed Power Conference, Albuquerque, NM, 1995, p. 607.

8. O. Sotolongo-Costa, Y. Moreno-Vega, J.J. LloverasGonzález, and J.C. Antoranz, Phys. Rev. Lett. 76, 42 (1996).

9. T. Kadono, Phys. Rev. Lett. 78, 1444 (1997).

10. J.B. Elliot, Ph. D. Thesis, Purdue University, 1995 (unpublished).

11. J.B. Elliot et al., (EOS Collaboration), Phys. Rev. C 62, 064603 (2000).

12. Dietrich Stauffer and Amnon Aharony, "Introduction to Percolation Theory," Taylor \& Francis Inc, 1992.

13. D. S. Sorenson et al., "Particle Distribution Measurements Using In-Line Fraunhofer Holography," in 22nd International Congress on High-Speed Photography and Photonics, Proc. SPIE 2869, 206-213 (1997). D.L. Paisley, A.M. Frank (eds.)

14. D.S. Sorenson et al., "In-Line Particle Field Holography at Pegasus," Proc. of the 10th IEEE Pulsed Power Conference, Albuquerque, NM, 1995, p. 1024.

15. Chandra S. Vikram, "Particle Field Holography," Cambridge Studies in Modern Optics (1992).

16 T. W. Tunnell, et. al, "Deriving particle distributions from in-line Fraunhofer holographic data", Proc. SPIE 3163, 558-565 (1997). 